\title{
Wie bei Kindern ein gutes Lungen-MRT gelingt
}

\author{
Sequenzen, technische Tipps und Perspektiven
}

Der Einsatz der MRT gewinnt in der pädiatrischen Lungenbildgebung weiter an Bedeutung. Lungen-MRT-Protokolle sind leider noch nicht standardisiert. Sie unterliegen individuellen Präferenzen und Erfahrungen. Daher sind die Ergebnisse der MRT-Diagnostik der Lunge von Einrichtung zu Einrichtung sehr unterschiedlich. Die Ergebnisse reichen von „diagnostisch unzureichend“ bis „exzellente diagnostische Qualität“. Die mangelnde Konsistenz der MRT-Untersuchungen ist eine große Hürde, die dazu beiträgt, dass die Lungen-MR noch nicht immer erste Wahl bei der Lungenbildgebung von Kindern ist.

Das Hauptproblem ist die Vermeidung von Bewegungsartefakten. Wir untersuchen die Lunge mit atemgetriggerten T2-Sequenzen. 3 Sequenzen ohne Kontrastmittel sind in der Regel ausreichend: T2-TSE transversal und koronar, T2-TSE mit Fettsättigung transversal. Die Daten werden dabei nur während der bewegungsfreien Exspiration erfasst. Das ist ein relevanter Unterschied zu Untersuchungen bei Erwachsenen, bei denen häufig T2-TSE-Multiple Breathhold Sequenzen in Inspiration angewendet werden. Dieses beeinflusst natürlich den Bildeindruck. Es ist entscheidend, dass die richtige TRZeit sowie die richtige Verzögerung gewählt wird. Die TR-Zeit beträgt maximal $2000 \mathrm{~ms}$, sie kann aber problemlos auf 500 ms redu- ziert werden, wenn eine hohe Atemfrequenz dies erfordert.

Wie beim CT werden die Arme über dem Kopf gehalten. Die korrekte Phasenkodierrichtung muss berücksichtigt werden: Anterior-posterior für transversale Sequenzen und head-feet für koronare Sequenzen. Die Schichtdicke beträgt höchstens $4 \mathrm{~mm}$ bei 1,5 Tesla-Geräten und 2,5-3,0 mm bei 3,0 Tesla-Geräten. Bei der Sedierung erschweren dorsale Atelektasen oft die Beurteilung von Lungenuntersuchungen. Mit Propofol zeigen bis zu $40 \%$ der Kinder dorsale Atelektasen. In solchen Fällen kann die Positionierung in die Bauchlage oder die Larynxmaskenbeatmung Abhilfe schaffen, sobald Atelektasen nach der ersten Sequenz sichtbar sind ( $\triangleright$ Abb. 1).

Einige typische MRT-Befunde werden demonstriert und die aktuell erreichbare Qualität wird anhand von Fallstudien diskutiert.

Viele Institutionen verwenden Atemhaltesequenzen (z. B. T2-HASTE, T1-GE mit Kontrast). Dies ist bei Kleinkindern schwierig, und die Bildqualität ist auch bei älteren Kindern und jungen Erwachsenen oft diagnostisch unzureichend bezüglich der Pathologie-Erkennung.

Eine neue Technik sind Lungen-Sequenzen mit ultrakurzer TE-Zeit (UTE-Sequenzen). Diese T1-gewichteten Sequenzen liefern Bilder, die denen von CT-Bildern (ohne

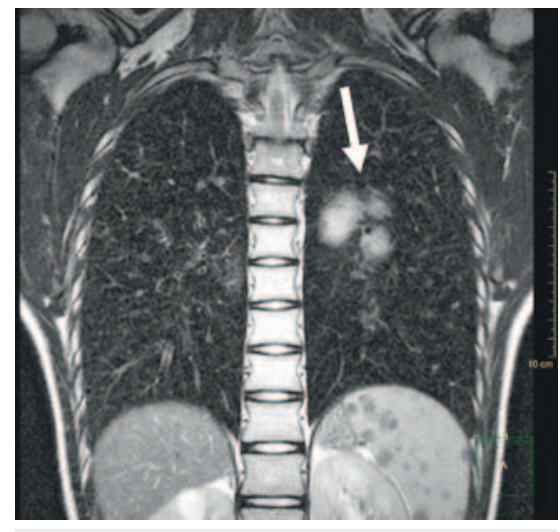

Abb. 1 Gute Bildqualität bei atemgetriggerter T2-TSE-Sequenz, Schichtbreite 2,5 mm, coronare Acquisition. Infiltrat im linken Oberlappen (Pfeil).

Kontrastmittel) ähnlich sind. Spezielle Ausleseverfahren und fortgeschrittene Triggertechniken (selfgated sequences) führen zu überzeugenden Bildern ohne Bewegungsartefakten. Man erhält Daten zum Teil mit Isovoxel-Abmessungen, zum Teil sogar im Submillimeter-Bereich. Obwohl diese Sequenzen zum großen Teil noch nicht als kommerzielles Produkt erhältlich sind, deutet sich hier eine substanzielle neue Qualität der Lungenbildgebung an, die die weitere Einführung der Lungen-MRT in der Kinderradiologie beflügeln wird ( $\triangleright \mathbf{A b b} .2)$. 

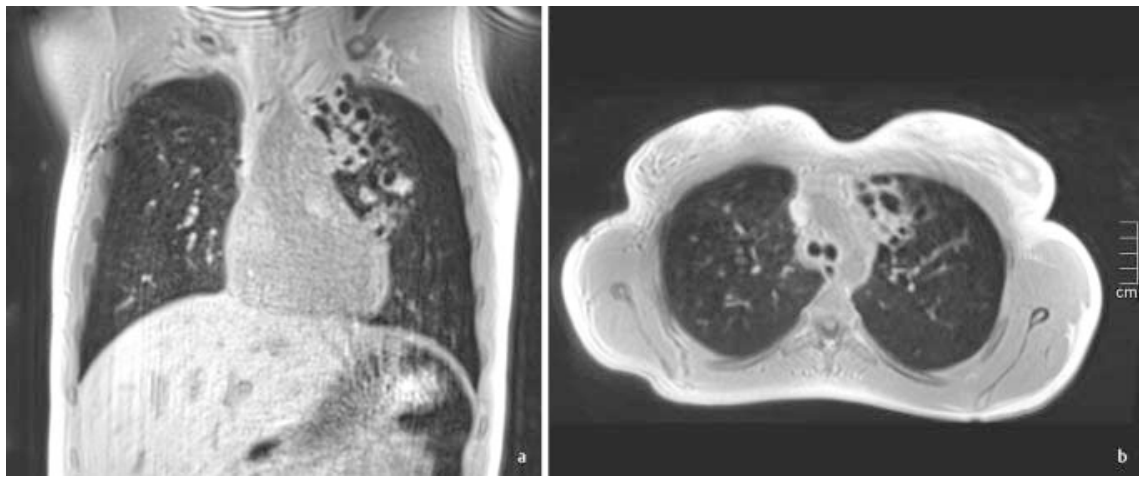

- Abb. 2 a T1-UTE-Sequenz, selfgated, koronare Acquisition. Man erhält ein CT-ähnliches Bild ohne Atemartefakte im Submillimeterbereich. Bronchiektasen im linken Oberlappen. b T1-UTE-Sequenz, selfgated, axiale Rekonstruktion aus dem original-koronaren Datensatz. Bronchiektasen im linken Oberlappen.

Interessenkonflikt

Die Autoren geben an, dass kein Interessenkonflikt besteht.

\section{Autorinnen/Autoren}

Franz Wolfgang Hirsch, Rebecca Anders, Christian Roth, Daniel Gräfe

Abteilung Kinderradiologie, Universität Leipzig
Korrespondenzadresse

Prof. Dr. Franz Wolfgang Hirsch

Abteilung Kinderradiologie, Universität

Leipzig

fw.hirsch@medizin.uni-leipzig.de

Bibliografie

DOI https://doi.org/10.1055/a-0943-1168 Online-Publikation: 2019

Fortschr Röntgenstr 2019; 191: S114-S115 (c) Georg Thieme Verlag KG, Stuttgart · New York ISSN 1433-5972 